\title{
Decisão Terapêutica e Metas
}

Coordenadora:

Frida Liane Plavnik (SP)

\section{Secretário:}

Antonio Felipe

Sanjuliani (RJ)

\section{Participantes:}

Abrão Cury (SP)

Antonio Carlos

Palandri Chagas (SP)

Antônio Felipe Simão

(SC)

Carlos Scherr (RJ)

Giovânio Vieira da

Silva (SP)

João Carlos Rocha (SP)

José Xavier de Mello

Filho (MA)

Marco A. Mota Gomes

(AL)

Maria Fátima de

Azevedo (RN)

Oswaldo Passarelli (SP)

Paulo César Veiga

Jardim (GO)

Rafael Leite Luna (RJ)

Sérgio Baiochi (GO)
A decisão terapêutica deve ser baseada no risco cardiovascular considerando-se a presença de fatores de risco, lesão em órgão-alvo e/ou doença cardiovascular estabelecida, e não apenas no nível da PA.

\section{Abordagem para Níveis de PA en- TRE 130-139/85-89 $\mathbf{m m H g}$}

A justificativa para intervenções em pessoas com esses níveis de PA baseia-se no fato de que metade do ônus atribuível ocorre para pressões sistólicas entre $130 \mathrm{e}$ $150 \mathrm{mmHg}$, isto é, incluindo o comportamento limítrofe de $\mathrm{PA}^{1}$ (Grau B).

Uma importante consideração a ser feita é que nessa faixa de PA há uma grande parcela de indivíduos com doenças cardiovascular e renal estabelecidas, além de boa parte de indivíduos com diabetes, doença renal, múltiplos fatores de risco cardiovascular e síndrome metabólica.

Assim, parece pertinente que esses indivíduos recebam tratamento medicamentoso. Deve-se, entretanto, considerar que foi demonstrado benefício dessa intervenção em estudo incluindo pacientes de alto risco com doença coronariana ${ }^{2}$ ou em outro que avaliou pacientes com doença encefálica prévia, ${ }^{3}$ e finalmente, em indivíduos diabéticos tipo 2. ${ }^{4}$ Alguns estudos aleatórios e controlados contra placebo sugerem que a instituição de tratamento medicamentoso em pacientes de alto risco reduz os eventos cardiovasculares quando há valores de PA entre 130-139/85-89 $\mathrm{mmHg}^{5-14}$ (Grau A).

\section{Abordagem em Pacientes Hiper- tensos com Risco Cardiovascular Médio, Alto e Muito Alto}

A instituição precoce do tratamento medicamentoso visa à proteção dos órgãos-alvos; redução do impacto causado pela elevação da PA, redução do impacto causado pela presença de fatores de risco associados e na progressão do processo aterosclerótico.

\section{Racional para Decisão Terapêutica}

Modificações de estilo de vida podem se refletir no retardo do desenvolvimento da HAS em indivíduos com pressão limítrofe. Têm também impacto favorável nos fatores de risco envolvidos no desenvolvimento ou agravo da hipertensão ${ }^{12-15}$ (Grau B).

Assim, devem ser indicadas indiscriminadamente.

O período de tempo recomendado para as medidas de modificação de estilo de vida isoladamente em pacientes hipertensos $\mathrm{e}$ naqueles com comportamento limítrofe da pressão arterial, com baixo risco cardiovascular, é de no máximo seis meses.

Caso os pacientes não estejam respondendo a essas medidas após três meses, uma nova avaliação, em seis meses, deve ser feita para confirmar o controle da PA. Se esse benefício não for confirmado, já está indicada a instituição do tratamento medicamentoso em associação.

Em pacientes com riscos médios, altos ou muito altos, independentemente da PA, a abordagem deve ser combinada (não medicamentosa e medicamentosa) para se atingir a meta preconizada o mais precocemente.

Para a decisão terapêutica, considerar a Tabela 1 e, para as metas de PA nas diferentes categorias de risco, considerar a Tabela 2.

Novos estudos não demostraram haver benefícios com reduções mais intensas da PA como se julgava anteriormente ${ }^{16,17}$ (Grau A). 


\section{Tabela 1 DECISÃO TERAPÊUTICA}

\begin{tabular}{ll} 
Categoria de risco & Considerar \\
\hline Rem risco adicional & $\begin{array}{l}\text { Tratamento não medicamentoso } \\
\text { isolado }\end{array}$ \\
\hline $\begin{array}{ll}\text { Risco adicional baixo } \\
\text { Tratamento não medicamentoso adicional médio, } \\
\text { isolado por até } 6 \text { meses. Se } \\
\text { não atingir a meta, associar } \\
\text { tratamento medicamentoso }\end{array}$ & $\begin{array}{l}\text { Tratamento não medicamentoso } \\
\text { + medicamentoso }\end{array}$
\end{tabular}

\section{Tabela 2 MetAS A SEREM ATINGIDAS EM CONFORMIDADE COM AS CARACTERÍSTICAS INDIVIDUAIS}

\begin{tabular}{lc} 
Categoria & \multicolumn{1}{c}{ Considerar } \\
\hline $\begin{array}{l}\text { Hipertensos estágios } 1 \text { e } 2 \text { com risco } \\
\text { cardiovascular baixo e médio }\end{array}$ & $<140 / 90 \mathrm{mmHg}$ \\
Hipertensos e comportamento limítrofe & \\
com risco cardiovascular alto e muito & \\
alto, ou com 3 ou mais fatores de risco, & $130 / 80 \mathrm{mmHg}$ \\
DM, SM ou LOA & \\
Hipertensos com insuficiência renal \\
com proteinúria > 1,0 g/L
\end{tabular}

(DM: diabetes melito; SM: síndrome metabólica; LOA: lesões em órgãos-alvo).

\section{Justificativa para as Metas de Pressão a SEREM ATINGIDAS}

\section{HIPERTENSÃO SISTÓLICA ISOLADA (HSI)}

Em pacientes com hipertensão sistólica isolada (HSI), a PA será dependente do risco cardiovascular, isto é, naqueles pacientes sem fator de risco adicional ou com risco cardiovascular baixo ou médio, a meta deve ser inferior a $140 \mathrm{mmHg}$. Já nos pacientes com risco cardiovascular alto ou muito alto, a meta deve ser igual a $130 \mathrm{mmHg}$.

\section{HIPERTROFIA VENTRICULAR ESQUERDA}

Tem sido observado que o controle rigoroso da PA sistólica $(130 \mathrm{mmHg})$ em pacientes hipertensos não diabéticos com pelo menos um fator de risco associado diminuiu a chance de hipertrofia ventricular esquerda, comparando-se com o controle não rigoroso $(\text { PAS }<140 \mathrm{mmHg})^{18}$ (Grau B).

\section{SíndROME METABÓLICA (SM)}

Embora não existam evidências quanto à indicação do tratamento medicamentoso em pacientes com SM e PA limítrofe, é importante frisar que essa condição está presente em cerca de $30 \%$ dos indivíduos com
HAS, sendo considerada um preditor independente de eventos cardiovasculares, mortalidade geral e cardiovascular (Grau A) $)^{19-21}$ (Grau C). ${ }^{22,23}$

O objetivo do tratamento é a redução do risco para eventos cardiovasculares, e, portanto, a presença de três ou mais fatores de risco ou lesões em órgãosalvo já sinaliza o alto risco para doença cardiovascular. ${ }^{21,22}$ Desta forma, pacientes com SM devem ser abordados de acordo com o risco cardiovascular.

\section{DIABÉTICOS}

Segundo as recomendações da American Diabetes Association - ADA de 2009, pacientes diabéticos com PA entre 130 e 139 mmHg e 80-89 mmHg podem ser tratados com modificação de estilo de vida por um tempo máximo de três meses. Se após esse período não houver resposta adequada, o tratamento medicamentoso deve ser iniciado.

Pacientes com PA $\geq 140 / 90 \mathrm{mmHg}$ no momento do diagnóstico ou durante o seguimento devem receber tratamento medicamentoso em conjunto com modificação do estilo de vida ${ }^{24}$ (Grau D). Sugere-se que a meta de PA seja 130/80 $\mathrm{mmHg}$, pois o estudo recentemente publicado não mostrou benefícios com reduções mais rigorosas da PA. ${ }^{16}$

\section{DOENÇA ARTERIAL PERIFÉRICA (DAP)}

Embora não haja consenso quanto à meta mais adequada em pacientes com DAP, em geral esses pacientes têm lesões em outros territórios vasculares, apresentando, portanto, alto risco cardiovascular.

\section{Curva J}

De acordo com revisão de Rosendorff e Black ${ }^{25}$ parece adequado evitar reduções importantes da PA diastólica (PAD) $(<65 \mathrm{mmHg})$ ou de forma muito rápida quando essa pressão inicial é muito elevada em pacientes com doença arterial coronariana oclusiva.

Inúmeros estudos documentaram uma relação inversa entre a PAD e a doença coronária. Em muitos deles os efeitos da "curva J" foram registrados em variações fisiológicas da $\mathrm{PAD}$, ou seja, abaixo de 70 a $80 \mathrm{mmHg}^{25}$ (Grau A). Para a mesma redução da PAD há pouca evidência do efeito "curva J" sobre outros órgãos-alvo, como cérebro e rim. Há também pouca evidência desse fenômeno envolvendo a PA sistólica. ${ }^{26}$

\section{REFERÊNCIAS}

1. Rodgers A, Ezzati M, Vander Hoorn S, Lopez AD, Lin RB, Murray CJ; Comparative Risk Assessment Collaborating Group. Distribution of major health risks: 
findings from the Global Burden of Disease study. PLoS Med 2004 Oct; 1(1):e27.

2. Heart Outcomes Prevention Evaluation Study Investigators. Effects of an angiotensin converting enzyme inhibitor, ramipril, on cardiovascular events in high risk patients. N Engl J Med 2000; 342:145-153.

3. PROGRESS Collaborative Group. Randomised trial of a perindopril based blood pressure lowering regimen among 6,105 individuals with previous stroke or transient ischaemic attack. Lancet 2001; 358:1033-1041.

4. ADVANCE Collaborative Group. Effects of a fixed combination of perindopril and indapamide on macrovascular and microvascular outcomes in patients with type 2 diabetes mellitus (the ADVANCE trial): a randomised controlled trial. Lancet 2007; 370:829-840.

5. The European Reduction of Cardiac Events with Perindopril in Stable Coronary Artery Disease Investigators. Efficacy of perindopril in reduction of cardiovascular events among patients with stable coronary artery disease: randomized, double-blind, placebocontrolled multicentre trial (EUROPA). Lancet 2003; 362:782-788.

6. Hansson L, Zanchetti A, Carruthers SG et al. Effects of intensive blood pressure lowering and low-dose aspirin in patients with hypertension: principal results of the Hypertension Optimal Treatment (HOT) randomised trial. HOT Study Group. Lancet 1998; 351:1755-1762.

7. Bangalore S, Messerli FH, Wun C et al. Treating to New Targets Steering Committee and Investigators. J-Curve revisited: an analysis of the Treating to New Targets (TNT) Trial. J Am Coll Cardiol 2009; 53:A217.8.

8. Weber MA, Julius S, Kjeldsen SE et al. Blood pressure dependent and ndependent effects of antihypertensive treatment on clinical events in the VALUE Trial. Lancet 2004; 363:2049-2051.

9. Mancia G, Messerli F, Bakris G, Zhou Q, Champion A, Pepine CJ. Blood pressure control and improved cardiovascular outcomes in the International Verapamil SRTrandolapril Study. Hypertension 2007; 50:299-305.

10. Sleight P, Redon J, Verdecchia P et al. ONTARGET investigators. Prognostic value of blood pressure in patients with high vascular risk in the Ongoing Telmisartan Alone and in combination with Ramipril Global Endpoint Trial study. J Hypertens 2009; 27:1360-1369.

11. Redon J, Sleight P, Mancia G et al. Safety and efficacy of aggressive blood pressure lowering among patients with diabetes: subgroup analyses from the ONTARGET trial. J Hypertens 2009; 27 (Suppl 4):S16.

12. Moore LL, Visioni AJ, Qureshi MM et al. Weight loss in overweight adults and the long-term risk of hypertension: the Framingham study. Arch Intern Med 2005; 165:1298-1303.

13. Halperin RO, Sesso HD, Ma J et al. Dyslipidemia and the risk of incident hypertension in men. Hypertension 2006; 47:45-50.
14. Haffner SM, Miettinen H, Gaskill SP, Stern MP. Metabolic precursors of hypertension. The San Antonio Heart Study. Arch Intern Med 1996; 156:1994-2001.

15. Zhang Y, Lee ET, Devereux RB et al. Prehypertension, diabetes, and cardiovascular disease risk in a population based sample: the Strong Heart Study. Hypertension 2006; 47:410- 414 .

16. The ACCORD Study Group. Effects of intensive bloodpressure control in type 2 Diabetes mellitus. N Engl J Med 2010. DOI:10.1056/NEJMoa1001286.

17. Pepine CJ, Handberg EM, Cooper-DeHoff RM et al., for the INVEST Investigators. A calcium antagonist vs. a non-calcium antagonist hypertension treatment strategy for patients with coronary artery disease. The International Verapamil-Trandolapril Study (INVEST): a randomized controlled trial. JAMA 2003; 290:2805-2816.

18. Verdecchia P, Staessen JA, Angeli F et al. On behalf of the Cardio-Sis investigators. Usual versus tight control of systolic blood pressure in non-diabetic patients with hypertension (Cardio-Sis): an open-label randomised trial. Lancet 2009; 374:525-533.

19. Schillaci G, Pirro M, Vaudo G et al. Prognostic value of the metabolic syndrome in essential hypertension. J Am Coll Cardiol 2004; 43:1817-1822.

20. Dekker JM, Girman C, Rhodes T et al. Metabolic syndrome and 10-year cardiovascular disease risk in the Hoorn Study. Circulation 2005; 112:666-673.

21. Mancia G, Bombelli M, Corrao G, Facchetti R, Madotto F, Giannattasio C et al. Metabolic syndrome in the Pressioni Arteriose Monitorate E Loro Associazioni (PAMELA) study: daily life blood pressure, cardiac damage, and prognosis. Hypertension 2007; 49:40-47.

22. Redon J, Cifkova R, Laurent S et al. On behalf of the Scientific Council of the European Society of Hypertension. The metabolic syndrome in hypertension: European society of hypertension position statement. J Hypertens 2008; 26:1891-1900.

23. Grundy SM, Cleeman JI, Daniels SR et al. Diagnosis and management of the metabolic syndrome. An American Heart Association/National Heart, Lung, and Blood Institute Scientific Statement. Circulation 2005; 112:2735-2752.

24. Standards of Medical Care in Diabetes - 2009. Position Statement. Diabetes Care 2009; 32, Suppl 1:S13-S61.

25. Rosendorff C, Black HR. Evidence for a lower target blood pressure for people with heart disease. Curr Opin Cardiol 2009; 24:318-324.

26. Messerli FH, Panjrath GS. J-Curve between blood pressure and coronary artery disease or essential hypertension: exactly how essential? J Am Coll Cardiol 2009; 54:1827-1834. 